\title{
Comparing the care needs of people living with and without HIV in Canadian home and long-term care settings
}

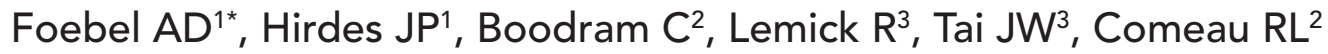

\begin{abstract}
Background: With the advent of highly active antiretroviral therapy (HAART), HIV has become a manageable chronic infection and individuals with it are living longer. Older individuals with HIV will begin to seek services across the continuum of health care. Whether their care needs differ from those who are HIV negative has not been well-characterized.
\end{abstract}

Objectives: To compare the demographic characteristics, chronic conditions, presence of infections, and mental health issues among HIV-positive versus HIV-negative individuals in home care, long-term care and complex continuing care settings across Canada.

Methods: This cross-sectional study used interRAl data to compare characteristics of HIV-positive and HIV-negative individuals in long-term care, complex continuing care and home care settings. Chi-square analyses explored differences between groups on co-infections, chronic disease and mental health issues.

Results: Data from 1,200,073 people were analyzed of whom 1,608 (0.13\%) had HIV. Overall, HIV-positive individuals had more co-infections but fewer chronic diseases than their HIV-negative counterparts. Depression, social isolation and the use of psychotropic medications were generally more prevalent in the HIV-positive cohort.

Conclusion: People living with HIV make up a small cohort of people with complex needs in home care and institutional settings and their care needs differ from those who are HIV negative. As HIV-positive people age, a better understanding of the context in which these issues are experienced will support appropriate interventions.

\section{Affiliations}

${ }^{1}$ School of Public Health and Health Systems, University of Waterloo, Waterloo, ON

${ }^{2}$ Centre for Communicable Diseases and Infection Control, Public Health Agency of Canada, Ottawa, ON

${ }^{3}$ Ontario Region, Public Health Agency of Canada, Toronto, ON

*Correspondence: adfoebel@ uwaterloo.ca

Suggested citation: Foebel AD, Hirdes JP, Boodram C, Lemrick R, Tai JW, Comeau RL. Comparing the care needs of people living with and without HIV in Canadian home and long-term settings. Can Comm Dis Rep 2016;42:52-6. https://doi.org/10.14745/ccdr.v42i03a01

\section{Introduction}

In many developed countries, advances in HIV management, namely highly active antiretroviral therapy (HAART), mean that HIV infection has become a chronic disease. For persons living with HIV-AIDS (PHAs), comorbidity profiles are shifting away from AIDS-defining illnesses toward HIV-associated non-AIDS illnesses such as cardiovascular and kidney diseases (1,2). Further, approximately two-thirds of older PHAs now live with other chronic conditions (3-11). The onset of these chronic conditions occurs earlier than in HIV-negative individuals $(1,5,11)$ and comorbidity includes more infections due to immune suppression and HAART $(1,2,12)$. Finally, despite advances in HAART, HIV-associated neurocognitive disorders are well-described long-term outcomes of HIV infection (13). This population likely differs greatly from younger PHAs and older adults without HIV, making the particular care needs of older PHAs unique.
HIV prevalence in Canada is expected to follow US projections, where it is estimated that half of HIV-positive individuals will be 50 years or older by 2050 (14). This demographic shift will have important implications for disease management and the choice of care setting for these individuals. It is likely that many adults with HIV will eventually seek support, treatment and care services in non-acute settings such as the home and long-term care (15). Whether these service providers are prepared to meet the unique care needs of these individuals is not known, but as individuals with HIV increasingly seek care options, pressure on such systems to respond will also increase.

interRAI standardized assessment data can help to establish whether care needs are being met across multiple health care settings in Canada. interRAl is an international not-for-profit research consortium initially established by clinicians and researchers with the goal of improving the quality of care and the quality of life in nursing homes in the USA

(www.interRAl.org) $(16,17)$. The interRAl assessment instruments 
were designed to provide a common, integrated approach to standardized assessment of vulnerable populations with complex care needs, such as those requiring home care and nursing home services $(16,18-20)$. The minimum data set version 2.0 (MDS 2.0) and interRAI Home Care (RAI-HC) instruments have been validated for use in the home care and long-term care settings, respectively (19-21). Although these instruments have been in use in Canada since 1996 and 2002, respectively, they have not been used to track the health care needs of HIV-positive individuals.

Currently, there is little evidence about the needs of HIV-positive individuals in home care or institutional environments. The objective of this study was to compare the care needs of people living with and without HIV in Canadian home care and long-term care settings, with a focus on co-infections, comorbidities and mental health issues.

\section{Methods}

\section{Settings}

This study included data from Canadian home care ( $\mathrm{HC})$, long-term care (LTC) and complex continuing care (CCC) settings, which are defined in the following text box.

\section{Definitions of care settings}

\begin{abstract}
Home care (HC): Services that include a mix of personal support, home nursing and some rehabilitative care that are provided in a client's home. Note: Long-stay clients are expected to be using services for 60 days or more.

Long-term care (LTC): Private, public and charitable nursing homes that provide regulated care to people with stable medical conditions who require 24 -hour care.

Complex continuing care (CCC): Hospitals or units in post-acute hospital settings that provide care to individuals with more severe impairments or more medically complex conditions and/or mental health needs than those who typically cared for in nursing homes. Note: These facilities are only available in Ontario and Manitoba.
\end{abstract}

Data from two Canadian interRAl assessment instruments were used to capture information about these three settings. Data for the home care sample were collected using the interRAl Home care (RAI-HC) instrument and data for the institutionalized samples (in LTC and CCC) came from the interRAI minimum data set version 2.0 (MDS 2.0) instrument. Data from these instruments were available from two national repositories managed by the Canadian Institute for Health Information (ClHI), the Home Care Reporting System (HCRS) and the Continuing Care Reporting System (CCRS) $(22,23)$. Data-sharing agreements between $\mathrm{ClHI}$, interRAI and the University of Waterloo allowed access to these data for this research. RAI-HC data were collected from 2002-2014 for all long-stay home care clients. For the LTC and CCC populations, MDS 2.0 assessments were collected between 1996 and 2014.

\section{Population}

All individuals in these settings were included in the study provided they had a completed interRAl assessment. $\mathrm{RAl}-\mathrm{HC}$ assessments from hospital settings were excluded as those assessments determine LTC eligibility and not home care planning.

The home care sample included individuals from British Columbia, Manitoba, Ontario, Nova Scotia and Yukon. The LTC sample included individuals from Alberta, British Columbia, Manitoba, New Brunswick, Newfoundland and Labrador, Nova Scotia, Ontario, Saskatchewan and Yukon. CCC data were available from Ontario and Manitoba.

\section{Measures}

interRAl instruments contain a comprehensive set of core items to describe client demographics and clinical characteristics that assist with care planning. Demographic information about age, sex and marital status (where "married" indicates married or common-law partners, including same-sex partners) are available from all interRAl records.

The measures included in this study comprised common chronic conditions associated with aging and conditions for which PHAs may be at higher risk (including infections and mental health comorbidities). Most data were available from both the RAI-HC and MDS 2.0 instruments. In both instruments, checklists of disease diagnoses are available for all of the chronic diseases (heart failure, emphysema/chronic obstructive pulmonary disease [COPD], diabetes, cancer, any psychiatric disorder and other cardiovascular diseases) and infectious diseases (pneumonia, tuberculosis and urinary tract infections [UTI]) assessed in this study. However, some infectious diseases (antibiotic-resistant infections, cellulitis, Clostridium difficile infection, conjunctivitis, respiratory infections, septicemia, sexually transmitted infections [STIs] and viral hepatitis) were only available from checklist items available on the MDS 2.0 and could not be measured in the home care sample.

For mental health symptoms (including anxiety, aggressive behaviour, hallucinations and social isolation), specific items record the presence or absence of the symptom on both the RAI-HC and the MDS 2.0. Depressive symptoms were examined using the Depression Rating Scale embedded within both interRAl assessment instruments; a score of three or higher on this scale indicated possible depression (24). The instruments also record the use of any psychotropic medications (antipsychotics, antidepressants, anxiolytics and sedatives) in the previous seven days.

\section{Analysis}

Differences in characteristics between HIV-positive and HIV-negative individuals in each care setting were tested using chi-square tests (significance level $p<0.05$ ) for all variables of interest. Age was collapsed into six categories to differentiate very young as well as very old individuals. All analyses were carried out using SAS version 9.4 (SAS Institute Inc., Cary, North Carolina). 


\section{Results}

The total sample comprised 1,200,073 people (Table 1). HIV prevalence was $0.13 \%$, which is slightly lower than that of the general Canadian population (0.21\% in 2014) (25); a large proportion of the sample came from Ontario (76\%). The long term care sample included 356,621 people; overall HIV prevalence was $0.05 \%$, but in 5 regions (New Brunswick, Newfoundland and Labrador, Nova Scotia, Saskatchewan and Yukon), there were no recorded HIV cases. The HIV prevalence

Table 1: Summary of study sample by care setting, Canada $(n=1,200,073)$

\begin{tabular}{|l|r|r|r|}
\hline Care setting & HIV/AIDS & \multicolumn{1}{|l|}{ Total } & \multicolumn{2}{|l|}{$\begin{array}{l}\text { HIV } \\
\text { prevalence } \\
(\%)\end{array}$} \\
\hline Long-term care $^{1}$ & 178 & 356,621 & 0.05 \\
\hline Complex continuing care $^{2}$ & 423 & 225,151 & 0.19 \\
\hline Home care $^{3}$ & 1007 & 618,301 & 0.16 \\
\hline TOTAL & 1608 & $1,200,073$ & 0.13 \\
\hline
\end{tabular}

Abbreviations: AIDS, acquired immunodeficiency syndrome; HIV, human immunodeficiency virus 'Sample from Alberta (10\%), British Columbia (17\%), Manitoba (4\%), New Brunswick $(<1 \%)$, Newfoundland $(<1 \%)$, Nova Scotia $(<1 \%)$, Ontario $(58 \%)$, Saskatchewan $(9 \%)$ and Yukon $(<1 \%)$ Sample from Manitoba (0.3\%) and Ontario (99.7\%) ${ }^{3}$ Sample from British Columbia (11\%), Manitoba (2\%), Nova Scotia $(8 \%)$, Ontario $(78 \%)$ and
Yukon $(<1 \%)$ rate in CCC was much higher at $0.19 \%$. Finally, the large home care sample $(n=618,301)$ had an overall HIV prevalence rate of $0.16 \%$, ranging from $0.07 \%$ in Nova Scotia to $0.49 \%$ in British Columbia.

Table 2 shows the demographic and diagnostic characteristics of the sample. The HIV-positive and HIV-negative groups differed on some key characteristics in the three care settings. Approximately $60 \%-65 \%$ of the people in the HIV-negative groups across the settings were female, compared with $20 \%-30 \%$ of people in the HIV-positive groups. Between $95 \%$ and $99 \%$ of HIV-negative people were over 50 years of age compared with $47 \%-72 \%$ of HIV-positive people. Marriage rates in the HIV-positive groups were half those of the HIV-negative groups, and men were less likely to be married than women, except in home care where both men and women with HIV were less likely to be married.

\section{Co-infections}

Disease diagnoses varied by care setting (Table 2). Infectious disease rates varied, but higher rates of pneumonia were seen in the HIV-positive groups in CCC and home care. The HIV-positive

Table 2: Demographic characteristics, co-infections, comorbid disease diagnoses and mental health characteristics of the Canadian sample, by care setting $(n=1,200,073)$

\begin{tabular}{|c|c|c|c|c|c|c|c|c|c|}
\hline \multirow[b]{2}{*}{ Characteristics } & \multicolumn{3}{|c|}{ Long-term care (\%) } & \multicolumn{3}{|c|}{ Complex continuing care (\%) } & \multicolumn{3}{|c|}{ Home care (\%) } \\
\hline & $\begin{array}{c}\text { Non HIV } \\
(n=356,443)\end{array}$ & $\begin{array}{l}\text { HIV } \\
178\end{array}$ & $p$ value & $\begin{array}{l}\text { Non HIV } \\
224,728\end{array}$ & $\begin{array}{l}\text { HIV } \\
423\end{array}$ & $p$ value & $\begin{array}{l}\text { Non HIV } \\
617,294 \\
\end{array}$ & $\begin{array}{c}\text { HIV } \\
1,007\end{array}$ & $p$ value \\
\hline Age (years) & & & $<0.0001$ & & & $<0.0001$ & & & $<0.0001$ \\
\hline $0-39$ & 0.3 & 5.6 & & 1.3 & 20.8 & & 2.2 & 11.1 & \\
\hline $40-49$ & 0.6 & 18.5 & & 2.3 & 31.9 & & 3.0 & 29.6 & \\
\hline $50-64$ & 4.2 & $43.3=$ & & 10.5 & 28.1 & & 11.6 & 37.5 & \\
\hline $65-74$ & 8.5 & 18.5 & & 18.0 & 9.5 & & 14.8 & 11.3 & \\
\hline $75-84$ & 28.0 & 10.7 & & 36.7 & 5.7 & & 33.0 & 6.3 & \\
\hline $85+$ & 58.4 & NR & & 31.4 & 4.0 & & 35.4 & 4.2 & \\
\hline Female & 65.8 & 22.5 & $<0.0001$ & 57.6 & 26.5 & $<0.0001$ & 62.8 & 30.3 & $<0.0001$ \\
\hline Married & 23.0 & 10.7 & $<0.0001$ & 39.2 & 18.0 & $<0.0001$ & 38.1 & 12.4 & $<0.0001$ \\
\hline Male & 39.3 & 9.4 & $<0.0001$ & 55.5 & 17.0 & $<0.0001$ & 56.5 & 11.6 & $<0.0001$ \\
\hline Female & 14.5 & 15.0 & 0.92 & 27.2 & 20.5 & 0.11 & 27.6 & 14.5 & $<0.0001$ \\
\hline \multicolumn{10}{|l|}{ Infections } \\
\hline Pneumonia & 2.6 & 3.9 & 0.28 & 7.3 & 13.2 & $<0.0001$ & 2.9 & 9.8 & $<0.0001$ \\
\hline Tuberculosis & 0.1 & NR & NR & 0.1 & 2.6 & $<0.0001$ & 0.1 & 4.1 & $<0.0001$ \\
\hline UTI & 7.0 & 6.7 & 0.88 & 16.5 & 9.5 & $<0.0001$ & 4.8 & 6.1 & 0.05 \\
\hline Antibiotic-resistant & 4.0 & 12.4 & $<0.0001$ & 6.7 & 9.9 & 0.007 & $n / a$ & $n / a$ & $n / a$ \\
\hline Cellulitis & 1.0 & NR & NR & 1.5 & 3.6 & 0.0005 & $\mathrm{n} / \mathrm{a}$ & $\mathrm{n} / \mathrm{a}$ & $n / a$ \\
\hline Clostridium difficile & 0.6 & NR & NR & 2.0 & 5.0 & $<0.0001$ & $n / a$ & $n / a$ & $n / a$ \\
\hline Conjunctivitis & 0.5 & 0 & 0.34 & 0.6 & 1.9 & 0.0003 & $\mathrm{n} / \mathrm{a}$ & $\mathrm{n} / \mathrm{a}$ & $\mathrm{n} / \mathrm{a}$ \\
\hline Respiratory infection & 2.7 & NR & NR & 3.6 & 4.5 & 0.3 & $n / a$ & $n / a$ & $n / a$ \\
\hline Septicemia & 0.6 & NR & NR & 1.4 & 4.5 & $<0.0001$ & $n / a$ & $n / a$ & $n / a$ \\
\hline STIs & 0.1 & 6.7 & $<0.0001$ & 0.1 & 12.1 & $<0.0001$ & $n / a$ & $n / a$ & $n / a$ \\
\hline Viral hepatitis & 1.2 & 23.6 & $<0.0001$ & 0.5 & 18.7 & $<0.0001$ & $\mathrm{n} / \mathrm{a}$ & $\mathrm{n} / \mathrm{a}$ & $n / a$ \\
\hline \multicolumn{10}{|l|}{ Diagnosis } \\
\hline Heart failure & 15.4 & 6.4 & 0.001 & 14.1 & 4.7 & $<0.0001$ & 13.0 & 6.1 & $<0.0001$ \\
\hline Emphysema/COPD & 17.1 & 18.6 & 0.61 & 18.3 & 14.7 & 0.05 & 17.7 & 19.8 & 0.09 \\
\hline Diabetes & 24.7 & 24.2 & 0.87 & 25.5 & 12.4 & $<0.0001$ & 25.2 & 15.6 & $<0.0001$ \\
\hline Cancer & 10.9 & 8.1 & 0.25 & 27.5 & 17.5 & $<0.0001$ & 17.7 & 12.5 & $<0.0001$ \\
\hline Any psychiatric & 53.4 & 52.8 & 0.87 & 40.6 & 48.0 & 0.002 & 30.0 & 46.2 & $<0.0001$ \\
\hline Other CVD & 66.1 & 32.6 & $<0.0001$ & 60.4 & 27.2 & $<0.0001$ & 67.4 & 32.2 & $<0.0001$ \\
\hline \multicolumn{10}{|l|}{ Mental health } \\
\hline Anxiety symptoms & 32.8 & 30.3 & 0.49 & 24.7 & 29.1 & 0.04 & 17.7 & 21.1 & 0.006 \\
\hline Anxiety disorders & 8.0 & 7.0 & 0.63 & 7.0 & 8.8 & 0.15 & $n / a$ & $n / a$ & $\mathrm{n} / \mathrm{a}$ \\
\hline Depression ${ }^{1}$ & 31.1 & 23.9 & 0.04 & 22.7 & 25.9 & 0.0008 & 18.1 & 25.7 & 0.004 \\
\hline Any aggressive behaviour & 43.9 & 48.3 & 0.23 & 27.1 & 33.6 & 0.003 & 9.9 & 7.9 & 0.03 \\
\hline Hallucinations/ Delusions & 5.9 & NR & 0.44 & 7.2 & 7.6 & 0.75 & 4.3 & 3.3 & 0.12 \\
\hline Social isolation & 4.9 & 9.1 & 0.09 & 6.3 & 13.4 & $<0.0001$ & 15.4 & 19.3 & 0.0006 \\
\hline \multicolumn{10}{|l|}{ Psychotropic drug use } \\
\hline Antipsychotics & 30.9 & 42.7 & 0.0006 & 19.7 & 27.0 & 0.0002 & 10.7 & 20.0 & $<0.0001$ \\
\hline Antidepressants & 46.8 & 58.4 & 0.002 & 29.1 & 33.6 & 0.04 & 24.9 & 36.3 & $<0.0001$ \\
\hline Anxiolytics & 14.7 & 22.5 & 0.003 & 29.8 & 39.5 & $<0.0001$ & 16.4 & 22.2 & $<0.0001$ \\
\hline Sedatives & 11.5 & 23.0 & $<0.0001$ & 15.5 & 15.6 & 0.94 & 20.9 & 25.2 & 0.0008 \\
\hline
\end{tabular}

Abbreviations: COPD, chronic obstructive pulmonary disease; CVD, cardiovascular disease; HIV, human immunodeficiency virus; STI, sexually transmitted infection;

UTI, urinary tract infection; NR, not reported (due to small cell size, $<9$ cases)

${ }^{1}$ Symptoms of probable depression defined as a score of $\geq 3$ on the Depression Rating Scale 
cohorts in all the settings had higher rates of tuberculosis infections. Rates of antibiotic-resistant infections, cellulitis and C. difficile were all higher in the HIV-positive cohort in the CCC and LTC settings. Septicemia prevalence was higher in the HIV-positive group in CCC, and STls were much more prevalent in the HIV-positive cohorts in both LTC $(6.7 \%$ vs. $0.1 \%)$ and CCC (12.1\% vs. $0.1 \%)$.

\section{Chronic disease diagnoses}

Chronic disease prevalence was generally lower among PHAs, with the exception of chronic psychiatric conditions, which were more common among HIV-positive individuals in home care $(p<0.0001)$ (Table 2). In the LTC setting, heart failure was lower in the HIV-positive group, but the rates of COPD, diabetes and cancer were similar between the HIV-positive and negative groups. In CCC and home care, the HIV-positive group had rates of COPD similar to the HIV-negative group but lower rates of heart failure, diabetes and cancer. Other cardiovascular diseases were significantly lower in the HIV-positive group in all three settings $(p<0.0001)$.

\section{Mental health issues}

Mental health issues were generally common and similar among LTC residents with and without HIV (Table 2). In CCC and home care, PHA had slightly higher rates of anxiety symptoms but these were not statistically significant. While aggressive behaviour was more prevalent in the HIV-positive cohort in CCC (33.6\% vs. $27.1 \%)$, it was less prevalent in the HIV-positive cohort in home care (7.9\% vs. 9.9\%). The HIV-positive group experienced significantly more social isolation than the HIV-negative group in the CCC and home care settings $(p<0.0001)$. Finally, across all care settings, rates of psychotropic drug use were higher in the HIV-positive versus the HIV-negative groups, but were not statistically significant for antidepressants and sedatives in the CCC setting.

\section{Discussion}

This is the largest study to date of PHAs living in care settings. It included 1,608 PHAs from across Canada and confirms work done in an earlier Ontario cohort study (26). Compared to their counterparts without HIV, older PHAs had more co-infections, fewer chronic diseases and a similar mental health profile, although they tended to use more psychotropic medications. With the exception of UTIs, co-infections were more common among PHAs in all care settings, consistent with previous research $(27,28)$. The rates of comorbid diseases varied among $\mathrm{PHAs}$, with lower overall prevalence than reported in earlier work (3-11). The younger age of PHA groups in this study may explain these findings, but we were not able to confirm this as the PHA sample size was not large enough to stratify by age.

This study also confirms the high prevalence of psychiatric diagnoses, depression and anxiety symptoms among older PHAs $(2,7,8,11,29-31)$. However, the prevalences of anxiety disorders and hallucinations or delusions were also quite high among the HIV-negative cohorts in these care settings. The prevalence of depression neared $25 \%$ among PHAs, which is slightly higher than rates of $21 \%$ in US nursing homes $(28,32)$.
The substantially higher rates of psychotropic medication use by HIV-positive groups appear to be a novel finding. Since this is a descriptive dataset, it is not possible to speculate on the appropriateness of this use. Rates of social isolation were higher among PHAs, especially in home care. Social isolation, known to be common among older PHAs, increases with age (3) and can lead to loneliness and depression (2).

Key strengths of this study are its inclusion of large samples from multiple sites and data from across Canada. These findings can be considered representative of the three care settings that were explored, as interRAl data are complete where mandated. Finally, these data allowed characterization of the specific needs of older PHAs as they begin to navigate care options in older age.

Nevertheless, the over-representation of data from Ontario and the variability of interRAl uptake across Canada are important limitations. Also, these findings cannot be compared to PHAs who do not access such care or who access AIDS/HIV-specific care from other care settings. Finally, this cross-sectional work could not determine causal relationships.

As the population ages, the demand for LTC to support, treat and care for older PHAs will increase. Failure to address the unique care needs of this group may lead to worse outcomes and increase the strain on health systems (33). The higher prevalence of psychotropic medication use among HIV-positive groups may be worthy of more attention, especially given the debate over the appropriateness of these therapies in general $(34,35)$. Monitoring of these trends is indicated.

Future research using a comprehensive national sample of older PHAs would provide information to regions excluded in the current study. Exploring policy options designed to improve both care and access in these settings should also be a priority, particularly in the home care setting where improved services could delay or prevent hospitalizations and LTC admissions.

\section{Conclusions}

More PHAs are living longer and experiencing HIV in the context of aging. Our findings suggest that PHAs in home care, LTC and CCC settings have major needs with respect to the management of co-infections, comorbidity and mental health issues that set them apart from their HIV-negative counterparts.

\section{Funding}

Drs. Foebel and Hirdes received funding from the Public Health Agency of Canada [contract \# 4500326437] to carry out a larger research project on which this manuscript is based.

\section{Conflict of interest}

All authors confirm that they have no conflicts of interest.

\section{References}

1. Deeks SG, Lewin SR, Havlir DV. The end of AIDS: HIV infection as a chronic disease. Lancet. 2013;382(9903):1525-33. 
2. High KP, Brennan-Ing M, Clifford DB, Cohen MH, Currier J, Deeks SG, et al. HIV and aging: state of knowledge and areas of critical need for research. A report to the NIH Office of AIDS Research by the HIV and Aging Working Group. J Acquir Immune Defic Syndr. 2012;60 Suppl 1:S1-18.

3. Greysen SR, Horwitz LI, Covinsky KE, Gordon K, Ohl ME, Justice $A C$. Does social isolation predict hospitalization and mortality among HIV+ and uninfected older veterans? J Am Geriatr Soc. 2013;61(9):1456-63.

4. Justice AC. HIV and aging: time for a new paradigm. Curr HIV/ AIDS Reports. 2010;7(2):69-76.

5. Onen NF, Overton ET, Seyfried W, Stumm ER, Snell M, Mondy $\mathrm{K}$, et al. Aging and HIV infection: a comparison between older HIV-infected persons and the general population. HIV Clin Trials. 2010;11(2):100-9.

6. Rodriguez-Penney AT, ludicello JE, Riggs PK, Doyle K, Ellis RJ, Letendre SL, et al. Co-morbidities in persons infected with HIV: increased burden with older age and negative effects on healthrelated quality of life. AIDS Patient Care STDs. 2013;27(1):5-16.

7. Balderson BH, Grothaus L, Harrison RG, McCoy K, Mahoney C, Catz S. Chronic illness burden and quality of life in an aging HIV population. AIDS Care. 2013;25(4):451-8.

8. Ball SC. The aging HIV population. Clin Pract. 2014;11(2):221321.

9. Brennan-Ing M, Seidel L, London AS, Cahill S, Karpiak SE. Service utilization among older adults with HIV: the joint association of sexual identity and gender. J Homosex. 2014;61(1):166-96.

10. Cardoso SW, Torres TS, Santini-Oliveira M, Marins LM, Veloso VG, Grinsztejn B. Aging with HIV: a practical review. Braz J Infect Dis. 2013;17(4):464-79.

11. Havlik RJ, Brennan M, Karpiak SE. Comorbidities and depression in older adults with HIV. Sex Health. 2011;8(4):551-9.

12. Deeks SG, Tracy R, Douek DC. Systemic effects of inflammation on health during chronic HIV infection. Immunity. 2013;39(4):63345.

13. Elbirt D, Mahlab-Guri K, Bezalel-Rosenberg S, Gill H, Attali M, Asher I. HIV-associated neurocognitive disorders (HAND). Israel Med Assoc J. 2015;17(1):54-9.

14. Cahill S, Darnell B, Guidry JA, Krivo-Kaufman A, Schaefer $\mathrm{N}$, Urbano L, et al. Growing older with the epidemic: HIV and aging. New York: Gay Men's Health Crisis; 2010 http:// hivandrehab.ca/EN/documents/a_pa_aging10_emb2.pdf

15. Wallach I, Brotman S. Ageing with HIV/AIDS: a scoping study among people aged 50 and over living in Quebec. Ageing Soc. 2013;33:1212-42.

16. Bernabei R, Gray L, Hirdes J, Pei X, Henrard JC, Jonsson PV, et al. International gerontology. In: High K, Halter J, Asthana S, Ouslander J, Tinetti M, Studenski S, Hazzard W, editors. Hazzard's Geriatric Medicine and Gerontology. 6th ed. New York: McGraw Medical; 2009. p. 69-96.

17. Morris JN, Hawes C, Fries BE, Phillips CD, Mor V, Katz S, et al. Designing the national resident assessment instrument for nursing homes. Gerontologist. 1990;30(3):293-307.

18. Hirdes JP, Fries BE, Morris JN, Steel K, Mor V, Frijters D, et al. Integrated health information systems based on the RAI/MDS series of instruments. Healthc Manage Forum. 1999;12(4):30-40.
19. Carpenter Gl. Accuracy, validity and reliability in assessment and in evaluation of services for older people: the role of the interRAl MDS assessment system. Age Ageing. 2006;35(4):327-9.

20. Hirdes JP, Ljunggren G, Morris JN, Frijters DH, Finne Soveri $H$, Gray L, et al. Reliability of the interRAl suite of assessment instruments: a 12-country study of an integrated health information system. BMC Health Serv Res. 2008;8:277.

21. Morris JN, Fries BE, Steel K, Ikegami N, Bernabei R, Carpenter $\mathrm{Gl}$, et al. Comprehensive clinical assessment in community setting: applicability of the MDS-HC. J Am Geriatr Soc. 1997;45(8):1017-24.

22. Canadian Institute for Health Information. Continuing care reporting system (CCRS) metadata . Ottawa (ON): The Institute; 2016. https://www.cihi.ca/en/types-of-care/hospital-care/ continuing-care/continuing-care-reporting-system-ccrs-metadata

23. Canadian Institute for Health Information. Home care reporting system (HCRS) Metadata: CIHI; 2016, https://www.cihi.ca/en/ types-of-care/community-care/home-care/hcrs-metadata

24. Burrows AB, Morris JN, Simon SE, Hirdes JP, Phillips C. Development of a minimum data set-based depression rating scale for use in nursing homes. Age Ageing. 2000;29(2):165-72.

25. Public Health Agency of Canada (PHAC). HIV/AIDS epi updates: national HIV prevalence and incidence estimates for 2011. Ottawa (ON): The Agency; 2014.

26. Foebel AD, Hirdes JP, Lemick R, Tai JW. Comparing the characteristics of people living with and without HIV in I ong-term care and home care in Ontario, Canada. AIDS Care. 2015;27(10):1343-53.

27. Stoff DM, Khalsa JH, Monjan A, Portegies P. Introduction: HIV/ AIDS and aging. AIDS. 2004;18 Suppl 1:S1-2.

28. Buchanan RJ, Wang S, Huang C. Profiles of nursing home residents with HIV. J Health Care Poor Underserved. 2002;13(3):379-91.

29. Dolder CR, Patterson TL, Jeste DV. HIV, psychosis and aging: past, present and future. AIDS. 2004;18 Suppl 1:S35-42.

30. Justice AC, McGinnis KA, Atkinson JH, Heaton RK, Young C, Sadek J, et al. Psychiatric and neurocognitive disorders among HIV-positive and negative veterans in care: Veterans Aging Cohort Five-Site Study. AIDS. 2004;18 Suppl 1:S49-59.

31. Public Health Agency of Canada (PHAC). Population-specific HIV/AIDS status report: people living with HIV/AIDS. Ottawa (ON): The Agency; 2013. http://www.phac-aspc.gc.ca/aids-sida/ publication/ps-pd/people-personnes/index-eng.php

32. Buchanan RJ, Wang $S$, Huang $C$. Analyses of nursing home residents with human immunodeficiency virus and depression using the minimum data set. AIDS Patient Care STDs. 2002;16(9):441-55.

33. Gough K, Karapita S. Facing the future together: An innovative response to the urgent HIV/AIDS crisis in Toronto. Toronto (ON): Casey House, 2011.

34. Counsell SR. 2015 Updated AGS Beers Criteria offer guide for safer medication use among older adults. J Gerontol Nurs. 2015;41(11):60-1.

35. Stevenson DG, Decker SL, Dwyer LL, Huskamp HA, Grabowski DC, Metzger ED, et al. Antipsychotic and benzodiazepine use among nursing home residents: findings from the 2004 National Nursing Home Survey. Am J Geriatr Psychiatry. 2010;18(12):1078-92. 